\title{
Shortest Path Problem Under Triangular Fuzzy Neutrosophic Information
}

\author{
Said Broumi \\ Laboratory of Information processing, Faculty of Science \\ Ben M’Sik, University Hassan II, B.P 7955, Sidi Othman, \\ Casablanca, Morocco \\ broumisaid78@gmail.com \\ Mohamed Talea \\ Laboratory of Information processing, Faculty of Science \\ Ben M'Sik, University Hassan II, B.P 7955, Sidi Othman, \\ Casablanca, Morocco \\ taleamohamed@yahoo.fr
}

\author{
Assia Bakali \\ Ecole Royale Navale, Boulevard Sour Jdid, B.P 16303 \\ Casablanca, Morocco. \\ assiabakali@yahoo.fr \\ Florentin Smarandache \\ Department of Mathematics, University of New \\ Mexico,705 Gurley Avenue, Gallup, NM 87301, USA \\ fsmarandache@gmail.com
}

\author{
Luige Vladareanu \\ Institute of Solid Mechanics, Romanian Academy, Bucharest, Romania. \\ luigiv2007@yahoo.com.sg, luigiv@imsar.bu.edu.ro
}

\begin{abstract}
In this paper, we develop a new approach to deal with neutrosphic shortest path problem in a network in which each edge weight (or length) is represented as triangular fuzzy neutrosophic number. The proposed algorithm also gives the shortest path length from source node to destination node using ranking function. Finally, an illustrative example is also included to demonstrate our proposed approach.
\end{abstract}

Keywords - triangular fuzzy neutrosophic sets; score function; Shortest path problem

\section{INTRODUCTION}

In 1995, Smarandache talked for the first time about neutrosophy and he in 1999 and 2005 [1, 2] defined one of the most important new mathematical tool which is an neutrosophic set theory for handling problems involving imprecise, incomplete, indeterminate and inconsistent information cannot be dealt with fuzzy sets as well as intuitionistic fuzzy sets Smarandache [3] introduced the concept of neutrosophic set and neutrosophic logic as generalization of the concepts of fuzzy sets [4], intuitionistic fuzzy sets [5]. The concept of neutrosophic set is characterized by three independent membership degrees namely truthmembership degree $(\mathrm{T})$, indeterminacy-membership degree (I), and falsity-membership degree (F). which are lies between nonstandard unit interval $]^{-} 0,1^{+}[$.

From scientific or engineering point of view, the neutrosophic set and set- theoretic operator will be difficult to apply in the real application. For this purpose, a subclass of the neutrosophic sets called single-valued neutrosophic sets (SVNS for short) was proposed by Wang et al [6]. The concept of single valued neutrosophic sets has caught attention to the researcher on various topics such as to be such as the decision making problem, medical diagnosis and so on. Later on, Smarandache extended the neutrosophic set to neutrosophic overset, underset, and offset [7]. Additional literature on neutrosophic sets can be found in $[7,8,9,10,11$, $12,13,14,15,16,17,18,19]$. However, in uncertain and complex situations, the truth-membership, the indeterminacymembership and the falsity-membership independently of SVNS cannot be represented with exact real numbers or interval numbers Moreover, triangular fuzzy number can handle effectively fuzzy data rather than interval number. For this purpose. Biswas et al. [20] proposed the concept of triangular fuzzy neutrosophic sets (TFNS) by combining triangular fuzzy numbers (TFNs) and single valued neutrosophic set (SVNS) and define some of its operational rules and developed triangular fuzzy neutrosophic number weighted arithmetic averaging and triangular fuzzy neutrosophic number weighted arithmetic geometric averaging operators to solve multi-attribute decision making problem . In TFNS the truth, indeterminacy and the falsity-membership functions are expressed with triangular fuzzy numbers instead of real numbers. Also, Ye [21] defined trapezoidal fuzzy neutrosophic set and developed trapezoidal fuzzy neutrosophic number weighted arithmetic averaging and trapezoidal fuzzy neutrosophic number weighted arithmetic geometric averaging operators to solve multi-attribute decision making problem. Recently, Broumi et al. [22, 23, 24] presented the concept of single valued neutrosophic graph, The single valued neutrosophic graph model allows the attachment of truth-membership (t), indeterminacymembership (i) and falsity- membership degrees (f) both to vertices and edges. The single valued neutrosophic graph is the generalization of fuzzy graph and intuitionistic fuzzy graph. In addition, Broumi et al. $[25,26]$ proposed the concept of interval valued neutrosophic graph as a generalization the 
concept of the fuzzy graph, intuitionistic fuzzy graph, interval valued fuzzy graph and single valued neutrosophic graph. Also, Broumi et al. [27, 28] proposed the concept of bipolar neutrosophic graph as a generalization the concept of the fuzzy graph, bipolar fuzzy graph, single valued neutrosophic graph. Smarandache [29, 30] proposed another variant of neutrosophic graphs based on literal indeterminacy .

In graph theory, the shortest path problem is the problem of finding a path between two nodes (or vertices) such that sum of the weight of its constituent edges is minimized. This problem has been studied for a long time and has attracted researchers from various areas of interests such operation research, computer science, communication network and various other problem. There are many shortest path problems [31-39] that have been studied with different types of input data, including fuzzy set, intuitionistic fuzzy sets, trapezoidal intuitionistic fuzzy sets vague set. Up to present, few research papers deal with shortest path in neutrosophic environment. Broumi et al. [40] proposed an algorithm for solving neutrosophic shortest path problem based on score function. The same authors in [41] proposed a study of neutrosphic shortest path with single valued trapezoidal neutrosophic number on a network. In addition Broumi et al. [42] proposed develop an algorithm to find the shortest path on a network in which the weights of the edges are represented by bipolar neutrosophic numbers. Motivated by the works done in [4042 ], in this paper a new method is proposed for solving shortest path problems in a network which the edges length are characterized by single valued triangular neutrosophic numbers.

The rest of the paper has been organized in the following way. In Section 2, a brief overview of neutrosophic sets, single valued neutrosophic sets and triangular fuzzy neutrosophic sets. In section 3, we give the network terminology. In Section 4, an algorithm is proposed for finding the shortest path and shortest distance in triangular fuzzy neutrosophic graph. In section 5 an illustrative example is provided to find the shortest path and shortest distance between the source node and destination node. Finally, in Section 6 we provide conclusion and proposal for further research

\section{PRELIMINARIES}

In this section we give the definition and some important results regarding neutrosophic sets, single valued neutrosophic sets and triangular fuzzy neutrosophic sets

Definition 2.1 [3]. Let $X$ be a space of points (objects) with generic elements in $\mathrm{X}$ denoted by $\mathrm{x}$; then the neutrosophic set $\mathrm{A}(\mathrm{NS} \mathrm{A})$ is an object having the form $\mathrm{A}=\left\{<\mathrm{x}: T_{A}(x)\right.$, $\left.I_{A}(x), F_{A}(x)>, \mathrm{x} \in \mathrm{X}\right\}$, where the functions $\mathrm{T}, \mathrm{I}, \mathrm{F}$ : $\mathrm{X} \rightarrow]^{-} 0,1^{+}$[define respectively the truth-membership function, an indeterminacy-membership function, and a falsitymembership function of the element $x \in X$ to the set $A$ with the condition:

$$
{ }^{-} 0 \leq T_{A}(x)+I_{A}(x)+F_{A}(x) \leq 3^{+} .
$$

The functions $T_{A}(x), I_{A}(x)$ and $F_{A}(x)$ are real standard or nonstandard subsets of $]^{-} 0,1^{+}[$.

Since it is difficult to apply NSs to practical problems, Wang et al. [14] introduced the concept of a SVNS, which is an instance of a NS and can be used in real scientific and engineering applications.

Definition 2.2 [4]. Let $X$ be a space of points (objects) with generic elements in $\mathrm{X}$ denoted by $\mathrm{x}$. A single valued neutrosophic set A (SVNS A) is characterized by truthmembership function $T_{A}(x)$, an indeterminacy-membership function $I_{A}(x)$, and a falsity-membership function $F_{A}(x)$. For each point $\mathrm{x}$ in $\mathrm{X} T_{A}(x), I_{A}(x), F_{A}(x) \in[0,1]$. A SVNS A can be written as

$$
\mathrm{A}=\left\{<\mathrm{x}: T_{A}(x), I_{A}(x), F_{A}(x)>, \mathrm{x} \in \mathrm{X}\right\}
$$

And for every $\mathrm{x} \in \mathrm{X}$

$$
0 \leq T_{A}(x)+I_{A}(x)+F_{A}(x) \leq 3 .
$$

Definition 2.3 [21]. Assume that $X$ be the finite universe of discourse and $\mathrm{F}[0,1]$ be the set of all triangular fuzzy numbers on $[0,1]$. A triangular fuzzy neutrosophic set (TFNNS) $\tilde{A}$ in $\mathrm{X}$ is represented

$$
\tilde{A}=\left\{<\mathrm{x}: \tilde{T}_{A}(x), \tilde{I}_{A}(x), \tilde{F}_{A}(x)>, \mathrm{x} \in \mathrm{X}\right\}
$$

Where $\tilde{T}_{A}(x): \mathrm{X} \rightarrow F[0,1], \quad \tilde{I}_{A}(x): \mathrm{X} \rightarrow F[0,1] \quad$ and $\tilde{F}_{A}(x): \mathrm{X} \rightarrow F[0,1]$. The triangular fuzzy numbers

$\tilde{T}_{A}(x)=\left(\mathrm{T}_{A}^{1}(x), \mathrm{T}_{A}^{2}(x), \mathrm{T}_{A}^{3}(x)\right)$,

$\tilde{I}_{A}(x)=\left(I_{A}^{1}(x), I_{A}^{2}(x), I_{A}^{3}(x)\right)$ and

$\tilde{F}_{A}(x)=\left(F_{A}^{1}(x), F_{A}^{2}(x), F_{A}^{3}(x)\right)$, respectively, denote the truth-membership, indeterminacy-membership and a falsitymembership degree of $\mathrm{x}$ in $\tilde{A}$ and for every $\mathrm{x} \in \mathrm{X}$

$$
0 \leq \mathrm{T}_{A}^{3}(x)+I_{A}^{3}(x)+F_{A}^{3}(x) \leq 3 .
$$

For notational convenience, the triangular fuzzy neutrosophic value (TFNV) $\tilde{A}$ is denoted by $\tilde{A}=\langle(a, b, c),(e, f, g),(r, s, t)\rangle$ where,

$\left(\mathrm{T}_{A}^{1}(x), \mathrm{T}_{A}^{2}(x), \mathrm{T}_{A}^{3}(x)\right)=(\mathrm{a}, \mathrm{b}, \mathrm{c})$,

$\left(I_{A}^{1}(x), I_{A}^{2}(x), I_{A}^{3}(x)\right)=(\mathrm{e}, \mathrm{f}, \mathrm{g})$, and

$\left(F_{A}^{1}(x), F_{A}^{2}(x), F_{A}^{3}(x)\right)=(\mathrm{r}, \mathrm{s}, \mathrm{t})$

Definition 2.4 [21]. A triangular fuzzy neutrosophic number $\tilde{A}=\langle(a, b, c),(e, f, g),(r, s, t)\rangle$ is said to be zero triangular fuzzy neutrosophic number if and only if $(\mathrm{a}, \mathrm{b}, \mathrm{c})=(0,0,0),(\mathrm{e}, \mathrm{f}, \mathrm{g})=(1,1,1)$ and $(\mathrm{r}, \mathrm{s}, \mathrm{t})=(1,1,1)$ 
Definition 2.5 [20]. Let $\tilde{A}_{1}=\left\langle\left(a_{1}, b_{1}, c_{1}\right),\left(e_{1}, f_{1}, g_{1}\right),\left(r_{1}, s_{1}, t_{1}\right)\right\rangle$ and $\tilde{A}_{2}=\left\langle\left(a_{2}, b_{2}, c_{2}\right),\left(e_{2}, f_{2}, g_{2}\right),\left(r_{2}, s_{2}, t_{2}\right)\right\rangle$ be two TFNVs in the set of real numbers. and $\lambda>0$. Then, the operations rules are defined as follows;

(i)

$\tilde{A}_{1} \oplus \tilde{A}_{2}=\left\langle\begin{array}{l}\left(a_{1}+a_{2}-a_{1} a_{2}, \mathrm{~b}_{1}+b_{2}-b_{1} b_{2}, c_{1}+c_{2}-c_{1} c_{2}\right), \\ \left(e_{1} e_{2}, f_{1} f_{2}, g_{1} g_{2}\right),\left(r_{1} r_{2}, s_{1} s_{2}, t_{1} t_{2}\right)\end{array}\right\rangle$

(ii)

$\tilde{A}_{1} \otimes \tilde{A}_{2}=\left\langle\begin{array}{l}\left(a_{1} a_{2}, b_{1} b_{2}, c_{1} c_{2}\right), \\ \left(e_{1}+e_{2}-e_{1} e_{2}, f_{1}+f_{2}-f_{1} f_{2}, g_{1}+g_{2}-g_{1} g_{2}\right), \\ \left(r_{1}+r_{2}-r_{1} r_{2}, s_{1}+s_{2}-s_{1} s_{2}, t_{1}+t_{2}-t_{1} t_{2}\right)\end{array}\right\rangle$

(iii) $\lambda \tilde{A}=\left\langle\begin{array}{l}\left(\left(1-\left(1-a_{1}\right)^{\lambda}, 1-\left(1-b_{1}\right)^{\lambda}, 1-\left(1-c_{1}\right)^{\lambda}\right)\right), \\ \left(\mathrm{e}_{1}^{\lambda}, f_{1}^{\lambda}, g_{1}^{\lambda}\right),\left(r_{1}^{\lambda}, \mathrm{s}_{1}^{\lambda},,_{1}^{\lambda}\right)\end{array}\right\rangle$

(iv) $\tilde{A}_{1}^{\lambda}=\left\langle\begin{array}{l}\left(a_{1}^{\lambda}, b_{1}^{\lambda}, c_{1}^{\lambda}\right), \\ \left(\left(1-\left(1-e_{1}\right)^{\lambda}, 1-\left(1-f_{1}\right)^{\lambda}, 1-\left(1-g_{1}\right)^{\lambda}\right)\right), \\ \left(\left(1-\left(1-r_{1}\right)^{\lambda}, 1-\left(1-s_{1}\right)^{\lambda}, 1-\left(1-t_{1}\right)^{\lambda}\right)\right)\end{array}\right)$

where $\lambda>0$

Ye [21] introduced the concept of score function and accuracy function. The score function $\mathrm{S}$ and the accuracy function $\mathrm{H}$ are applied to compare the grades of TFNS. These functions shows that greater is the value, the greater is the TFNS and by using these concept paths can be ranked.

Definition 2.6. Let $\tilde{A}_{1}=\left\langle\left(a_{1}, b_{1}, c_{1}\right),\left(e_{1}, f_{1}, g_{1}\right),\left(r_{1}, s_{1}, t_{1}\right)\right\rangle$ be a TFNV, then, the score function $S\left(\tilde{A}_{1}\right)$ and an accuracy function $H\left(\tilde{A}_{1}\right)$ of TFNV are defined as follows:

(i)

$s\left(\tilde{A}_{1}\right)=\frac{1}{12}\left[8+\left(a_{1}+2 b_{1}+c_{1}\right)-\left(e_{1}+2 f_{1}+g_{1}\right)-\left(r_{1}+2 s_{1}+t_{1}\right)\right]$

(ii) $H\left(\tilde{A}_{1}\right)=\frac{1}{4}\left[\left(a_{1}+2 b_{1}+c_{1}\right)-\left(r_{1}+2 s_{1}+t_{1}\right)\right]$

In order to make a comparisons between two TFNV, Ye [21], presented the order relations between two TFNVs.

Definition 2.7. Let $\tilde{A}_{1}=\left\langle\left(a_{1}, b_{1}, c_{1}\right),\left(e_{1}, f_{1}, g_{1}\right),\left(r_{1}, s_{1}, t_{1}\right)\right\rangle$ and $\tilde{A}_{2}=\left\langle\left(a_{2}, b_{2}, c_{2}\right),\left(e_{2}, f_{2}, g_{2}\right),\left(r_{2}, s_{2}, t_{2}\right)\right\rangle$ be two TFNVs in the set of real numbers. Then, we define a ranking method as follows:

i. If $s\left(\tilde{A}_{1}\right) \succ s\left(\tilde{A}_{2}\right)$, then $\tilde{A}_{1}$ is greater than $\tilde{A}_{2}$, that is, $\tilde{A}_{1}$ is superior to $\tilde{A}_{2}$, denoted by $\tilde{A}_{1} \succ \tilde{A}_{2}$ ii. If $s\left(\tilde{A}_{1}\right)=s\left(\tilde{A}_{2}\right)$, and $H\left(\tilde{A}_{1}\right) \succ H\left(\tilde{A}_{2}\right)$ then $\tilde{A}_{1}$ is greater than $\tilde{A}_{2}$, that is, $\tilde{A}_{1}$ is superior to $\tilde{A}_{2}$, denoted by $\tilde{A}_{1} \succ \tilde{A}_{2}$.

\section{NETWORK TERMINOLOGY}

Consider a directed network $\mathrm{G}(\mathrm{V}, \mathrm{E})$ consisting of a finite set of nodes $\mathrm{V}=\{1,2, \ldots, \mathrm{n}\}$ and $\mathrm{a}$ set of $\mathrm{m}$ directed edges $\mathrm{E} \subseteq \mathrm{VxV}$. Each edge is denoted is denoted by an ordered pair $(\mathrm{i}, \mathrm{j})$ where $\mathrm{i}, \mathrm{j} \in \mathrm{V}$ and $i \neq j$. In this network, we specify two nodes, denoted by $\mathrm{s}$ and $\mathrm{t}$, which are the source node and the destination node, respectively. We define a path $P_{i j}=\{\mathrm{i}$ $\left.=i_{1},\left(i_{1}, i_{2}\right), i_{2}, \ldots, i_{l-1},\left(i_{l-1}, i_{l}\right), i_{l}=\mathrm{j}\right\}$ of alternating nodes and edges. The existence of at least one path $P_{s i}$ in G $(\mathrm{V}, \mathrm{E})$ is assumed for every $\mathrm{i} \in \mathrm{V}-\{\mathrm{s}\}$.

$d_{i j}$ denotes triangular fuzzy neutrosophic number associated with the edge (i, j), corresponding to the length necessary to traverse $(i, j)$ from $i$ to $j$. the neutrosophic distance along the path $\mathrm{P}$ is denoted as $\mathrm{d}(\mathrm{P})$ is defined as

$$
\mathrm{d}(\mathrm{P})=\sum_{(\mathrm{i}, \mathrm{j} \in \mathrm{P})} d_{i j}
$$

Remark : A node $i$ is said to be predecessor node of node $j$ if

(i) Node $\mathrm{i}$ is directly connected to node $\mathrm{j}$.

(ii) The direction of path connecting node $i$ and $j$ from $i$ to $j$.

III. TRIANGULAR FUZZY NEUTROSOPHIC PATH

PROBLEM

In this paper the edge length in a network is considered to be a neutrosophic number, namely, triangular fuzzy neutrosophic number.

The algorithm for the shortest path proceeds in 6 steps.

Step 1 Assume $\tilde{d}_{1}=<(0,0,0),(1,1,1),(1,1,1)>$ and label

the source node (say node1) as $\left[\tilde{d}_{1}=<(0,0,0),(1,1,1),(1,1\right.$, 1)>,-].

Step 2 Find $\tilde{d}_{j}=\operatorname{minimum}\left\{\tilde{d}_{i} \oplus \tilde{d}_{i j}\right\} ; \mathrm{j}=2,3, \ldots, \mathrm{n}$.

Step 3 If minimum occurs corresponding to unique value of $i$ i.e., $\mathrm{i}=\mathrm{r}$ then label node $\mathrm{j}$ as $\left[\tilde{d}_{j}, \mathrm{r}\right]$. If minimum occurs corresponding to more than one values of $i$ then it represents that there are more than one interval valued neutrosophic path between source node and node $\mathrm{j}$ but triangular fuzzy neutrosophic distance along path is $\tilde{d}_{j}$, so choose any value of i.

Step 4 Let the destination node (node n) be labeled as $\left[\tilde{d}_{n}, l\right]$, then the triangular fuzzy neutrosophic shortest distance between source node is $\tilde{d}_{n}$.

Step 5 Since destination node is labeled as $\left[\tilde{d}_{n}, l\right]$, so, to find the triangular fuzzy neutrosophic shortest path between source node and destination node, check the label of node 1 . Let it be 
$\left[\tilde{d}_{l}, \mathrm{p}\right]$, now check the label of node $\mathrm{p}$ and so on. Repeat the same procedure until node 1 is obtained.

Step 6 Now the triangular fuzzy neutrosophic shortest path can be obtained by combining all the nodes obtained by the step 5.

Remark 5.1 Let $\tilde{A}_{i} ; \mathrm{i}=1,2, \ldots, \mathrm{n}$ be a set of triangular fuzzy neutrosophic numbers, if $\mathrm{S}\left(\tilde{A}_{k}\right)<\mathrm{S}\left(\tilde{A}_{i}\right)$, for all i, the triangular fuzzy neutrosophic number is the minimum of $\tilde{A}_{k}$

\section{ILLUSTRATIVE EXAMPLE}

In order to illustrate the above procedure consider a small example network shown in Fig. 2, where each arc length is represented as triangular fuzzy neutrosophic number as shown in Table 2. The problem is to find the shortest distance and shortest path between source node and destination node on the network.

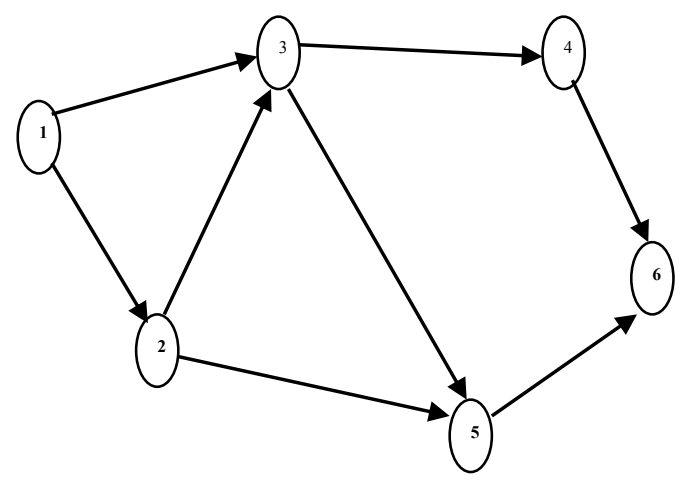

Fig.1 A network with triangular fuzzy neutrosophic edges

In this network each edge have been assigned to triangular fuzzy neutrosophic number as follows:

\begin{tabular}{|l|c|}
\hline Edges & triangular fuzzy neutrosophic distance \\
\hline $1-2$ & $<(0.1,0.2,0.3),(0.2,0.3,0.5),(0.4,0.5,0.6)>$ \\
\hline $1-3$ & $<(0.2,0.4,0.5),(0.3,0.5,0.6),(0.1,0.2,0.3)>$ \\
\hline $2-3$ & $<(0.3,0.4,0.6),(0.1,0.2,0.3),(0.3,0.5,0.7)>$ \\
\hline $2-5$ & $<(0.1,0.3,0.4),(0.3,0.4,0.5),(0.2,0.3,0.6)>$ \\
\hline $3-4$ & $<(0.2,0.3,0.5),(0.2,0.5,0.6),(0.4,0.5,0.6]>$ \\
\hline $3-5$ & $<(0.3,0.6,0.7),(0.1,0.2,0.3),(0.1,0.4,0.5)>$ \\
\hline $4-6$ & $<(0.4,0.6,0.8),(0.2,0.4,0.5),(0.1,0.3,0.4)>$ \\
\hline $5-6$ & $<(0.2,0.3,0.4),(0.3,0.4,0.5),(0.1,0.3,0.5)>$ \\
\hline
\end{tabular}

graphs

Table 1. weights of the triangular fuzzy neutrosophic

Solution since node 6 is the destination node, so $\mathrm{n}=6$.

assume $\tilde{d}_{1}=<(0,0,0),(1,1,1),(1,1,1)>$ and label the source node ( say node 1$)$ as $[<(0,0,0),(1,1,1),(1,1,1)>,-]$, the value of $\tilde{d}_{j} ; \mathrm{j}=2,3,4,5,6$ can be obtained as follows:
Iteration 1 Since only node 1 is the predecessor node of node 2 , so putting $i=1$ and $j=2$ in step 2 of the proposed algorithm, the value of $\tilde{d}_{2}$ is

$\tilde{d}_{2}=\operatorname{minimum}\left\{\tilde{d}_{1} \oplus \tilde{d}_{12}\right\}=\operatorname{minimum}\{<(0,0,0),(1,1,1),(1$, $1,1)\rangle \oplus\langle(0.1,0.2,0.3),(0.2,0.3,0.5),(0.4,0.5,0.6)\rangle=$ $<(0.1,0.2,0.3),(0.2,0.3,0.5),(0.4,0.5,0.6)>$

Since minimum occurs corresponding to $i=1$, so label node 2 as $[<(0.1,0.2,0.3),(0.2,0.3,0.5),(0.4,0.5,0.6)>, 1]$ $\tilde{d}_{2}=\langle(0.1,0.2,0.3),(0.2,0.3,0.5),(0.4,0.5,0.6)>$

Iteration 2 The predecessor node of node 3 are node 1 and node 2 , so putting $i=1,2$ and $j=3$ in step 2 of the proposed algorithm, the value of $\tilde{d}_{3}$ is $\tilde{d}_{3}=$

$\operatorname{minimum}\left\{\tilde{d}_{1} \oplus \tilde{d}_{13}, \tilde{d}_{2} \oplus \tilde{d}_{23}\right\}=\operatorname{minimum}\{<(0,0,0),(1,1,1)$, $(1,1,1)>\oplus<(0.2,0.4,0.5),(0.3,0.5,0.6),(0.1,0.2,0.3)>$, $<(0.1,0.2,0.3),(0.2,0.3,0.5),(0.4,0.5,0.6)>\oplus<(0.3,0.4$, $0.6),(0.1,0.2,0.3),(0.3,0.5,0.7)>\}=\operatorname{minimum}\{<(0.2,0.4$, $0.5),(0.3,0.5,0.6),(0.1,0.2,0.3)>,<(0.37,0.52,0.72)$, $(0.02,0.06,0.15),(0.12,0.25,0.42)>\}$

$\mathrm{S}(<(0.2,0.4,0.5),(0.3,0.5,0.6),(0.1,0.2,0.3)>)$

$s\left(\tilde{A}_{1}\right)=\frac{1}{12}\left[8+\left(a_{1}+2 b_{1}+c_{1}\right)-\left(e_{1}+2 f_{1}+g_{1}\right)-\left(r_{1}+2 s_{1}+t_{1}\right)\right]=0.57$ $\mathrm{S}(<(0.37,0.52,0.72),(0.02,0.06,0.15),(0.12,0.25,0.42)>)=$ 0.73

Since $S(<(0.2,0.4,0.5),(0.3,0.5,0.6),(0.1,0.2,0.3)>)<$ $\mathrm{S}(<(0.37,0.52,0.72),(0.02,0.06,0.15),(0.12,0.25,0.42)>)$ So, minimum $\{<(0.2,0.4,0.5),(0.3,0.5,0.6),(0.1,0.2,0.3)>$, $<(0.37,0.52,0.72),(0.02,0.06,0.15),(0.12,0.25,0.42)>\}$ $=<(0.2,0.4,0.5),(0.3,0.5,0.6),(0.1,0.2,0.3)>$

Since minimum occurs corresponding to $i=1$, so label node 3 as $[<(0.2,0.4,0.5),(0.3,0.5,0.6),(0.1,0.2,0.3)>, 1]$ $\tilde{d}_{3}=<(0.2,0.4,0.5),(0.3,0.5,0.6),(0.1,0.2,0.3)>$

Iteration 3. The predecessor node of node 4 is node 3 , so putting $\mathrm{i}=3$ and $\mathrm{j}=4$ in step 2 of the proposed algorithm, the value of $\tilde{d}_{4}$ is $\tilde{d}_{4}=\operatorname{minimum}\left\{\tilde{d}_{3} \oplus \tilde{d}_{34}\right\}=\operatorname{minimum}\{<(0.2$, $0.4,0.5),(0.3,0.5,0.6),(0.1,0.2,0.3)>$

$\oplus<(0.2,0.3,0.5),(0.2,0.5,0.6),(0.4,0.5,0.6]>\}=<(0.36$, $0.58,0.75),(0.06,0.25,0.36),(0.04,0.1,0.18)>$

So minimum $\{<(0.2,0.4,0.5),(0.3,0.5,0.6),(0.1,0.2,0.3)>$ $\oplus<(0.2,0.3,0.5),(0.2,0.5,0.6),(0.4,0.5,0.6]>\}=<(0.36$, $0.58,0.75),(0.06,0.25,0.36),(0.04,0.1,0.18)>$

Since minimum occurs corresponding to $\mathrm{i}=3$, so label node 4 as $[<(0.36,0.58,0.75),(0.06,0.25,0.36),(0.04,0.1,0.18)>$ ,3]

$\tilde{d}_{4}=<(0.36,0.58,0.75),(0.06,0.25,0.36),(0.04,0.1,0.18)$

Iteration 4 The predecessor node of node 5 are node 2 and node 3 , so putting $\mathrm{i}=2,3$ and $\mathrm{j}=5$ in step 2 of the proposed algorithm, the value of $\tilde{d}_{5}$ is $\tilde{d}_{5}=$ $\operatorname{minimum}\left\{\tilde{d}_{2} \oplus \tilde{d}_{25}, \tilde{d}_{3} \oplus \tilde{d}_{35}\right\}=\operatorname{minimum}\{<(0.1,0.2,0.3)$, $(0.2,0.3,0.5),(0.4,0.5,0.6)>\oplus<(0.1,0.3,0.4),(0.3,0.4$, 
$0.5),(0.2,0.3,0.6)>, \quad<(0.2,0.4,0.5),(0.3,0.5,0.6),(0.1,0.2$ $0.3)>\oplus<(0.3,0.6,0.7),(0.1,0.2,0.3),(0.1,0.4,0.5)>\}=$ Minimum $\{<(0.19,0.44,0.58),(0.06,0.12,0.25),(0.02,0.06$, $0.18)>,<(0.44,0.76,0.85),(0.03,0.1,0.18),(0.01,0.08$, $0.15)>$ \}

$\mathrm{S}(<(0.19,0.44,0.58),(0.06,0.12,0.25),(0.02,0.06,0.18)>)=$ 0.73

$\mathrm{S}(<(0.44,0.76,0.85),(0.03,0.1,0.18),(0.01,0.08,0.15)>)=$ 0.84

Since $S(<(0.19,0.44,0.58),(0.06,0.12,0.25),(0.02,0.06$, $0.18)>)<\mathrm{S}(<(0.44,0.76,0.85),(0.03,0.1,0.18),(0.01,0.08$, $0.15)>$ )

Minimum $\{<(0.19,0.44,0.58),(0.06,0.12,0.25),(0.02,0.06$, $0.18)>,<(0.44,0.76,0.85),(0.03,0.1,0.18),(0.01,0.08$,

$0.15)>$ \}

$=<(0.19,0.44,0.58),(0.06,0.12,0.25),(0.02,0.06,0.18)>$

$\tilde{d}_{5}=<(0.19,0.44,0.58),(0.06,0.12,0.25),(0.02,0.06,0.18)>$

Since minimum occurs corresponding to $\mathrm{i}=2$, so label node 5 as $[<(0.19,0.44,0.58),(0.06,0.12,0.25),(0.02,0.06,0.18)>$, 2]

Iteration 5. The predecessor node of node 6 are node 4 and node 5 , so putting $\mathrm{i}=4,5$ and $\mathrm{j}=6$ in step 2 of the proposed algorithm, the value of $\tilde{d}_{6}$ is $\tilde{d}_{6}=$

$\operatorname{minimum}\left\{\tilde{d}_{4} \oplus \tilde{d}_{46}, \tilde{d}_{5} \oplus \tilde{d}_{56}\right\}=\operatorname{minimum}\{<(0.36,0.58$, $0.75),(0.06,0.25,0.36),(0.04,0.1,0.18)>\oplus<(0.4,0.6,0.8)$, $(0.2,0.4,0.5),(0.1,0.3,0.4)>,<(0.19,0.44,0.58),(0.06$, $0.12,0.25),(0.02,0.06,0.18)>\oplus<(0.2,0.3,0.4),(0.3,0.4$, $0.5),(0.1,0.3,0.5)>\}=\operatorname{minimum}\{<(0.616,0.832,0.95)$, $(0.012,0.1,0.18),(0.004,0.03,0.072)>,<(0.352,0.608$, $0.748),(0.018,0.048,0.125),(0.002,0.018,0.09)>$ \}

$\mathrm{S}(<(0.616,0.832,0.95),(0.012,0.1,0.18),(0.004,0.03$, $0.072)>)=0.89$

$\mathrm{S}(<(0.352,0.608,0.748),(0.018,0.048,0.125),(0.002,0.018$, $0.09)>)=0.83$

Since S $(<(0.352,0.608,0.748),(0.018,0.048,0.125),(0.002$, $0.018,0.09)>)<\mathrm{S}(<(0.616,0.832,0.95),(0.012,0.1,0.18)$, $(0.004,0.03,0.072)>)$

minimum $\{<(0.616,0.832,0.95),(0.012,0.1,0.18),(0.004$, $0.03,0.072)>,<(0.352,0.608,0.748),(0.018,0.048,0.125)$, $(0.002,0.03,0.054)>\}=<(0.352,0.608,0.748),(0.018,0.048$, $0.125),(0.002,0.018,0.09)>$

$\tilde{d}_{6}=<(0.352,0.608,0.748),(0.018,0.048,0.125),(0.002$,

$0.018,0.09)>$

Since minimum occurs corresponding to $i=5$, so label node 6 as $[<(0.352,0.608,0.748),(0.018,0.048,0.125),(0.002$, $0.018,0.09)>, 5]$

$\tilde{d}_{6}=<(0.352,0.608,0.748),(0.018,0.048,0.125),(0.002$,

$0.018,0.09)>$

Since node 6 is the destination node of the given network, so the triangular fuzzy neutrosophic shortest distance between node 1 and node 6 is $<(0.352,0.608,0.748),(0.018,0.048$, $0.125),(0.002,0.018,0.09)>$

Now the triangular fuzzy neutrosophic shortest path between node 1 and node 6 can be obtained by using the following procedure:
Since node 6 is labeled by $[<(0.352,0.608,0.748),(0.018$, $0.048,0.125),(0.002,0.018,0.09)>, 5]$, which represents that we are coming from node 5 . Node 5 is labeled by $[<(0.19$, $0.44,0.58),(0.06,0.12,0.25),(0.02,0.06,0.18)>, 2]$, which represents that we are coming from node 2 . Node 2 is labeled by $[<(0.1,0.2,0.3),(0.2,0.3,0.5),(0.4,0.5,0.6)>, 1]$ which represents that we are coming from node 1 . Now the triangular fuzzy neutrosophic shortest path between node 1 and node 6 is obtaining by joining all the obtained nodes. Hence the triangular fuzzy neutrosophic shortest path is $1 \rightarrow 2 \rightarrow 5 \rightarrow 6$ The triangular fuzzy neutrosophic shortest distance and the neutrosophic shortest path of all nodes from node 1 is shown in the table 2 and the labeling of each node is shown in figure 4

\begin{tabular}{|l|l|l|}
\hline $\begin{array}{l}\mathrm{N} \\
\mathrm{o} \\
\mathrm{de}\end{array}$ & \multicolumn{1}{|c|}{$\tilde{d}_{i}$} & \multicolumn{1}{|c|}{$\begin{array}{c}\text { Triangular fuzzy } \\
\text { neutrosophic } \\
\text { shortest path } \\
\text { between jth and } \\
\text { 1st node }\end{array}$} \\
\hline 2 & $\begin{array}{l}<(0.1,0.2,0.3),(0.2,0.3,0.5),(0.4, \\
0.5,0.6)>\end{array}$ & $1 \rightarrow 2$ \\
\hline 3 & $\begin{array}{l}<(0.2,0.4,0.5),(0.3,0.5,0.6),(0.1, \\
0.2,0.3)>\end{array}$ & $1 \rightarrow 3$ \\
\hline 4 & $\begin{array}{l}<(0.36,0.58,0.75),(0.06,0.25,0.36), \\
(0.04,0.1,0.18)>\end{array}$ & $1 \rightarrow 3 \rightarrow 4$ \\
\hline 5 & $\begin{array}{l}<(0.19,0.44,0.58),(0.06,0.12,0.25), \\
(0.02,0.06,0.18)>\end{array}$ & $1 \rightarrow 2 \rightarrow 5$ \\
\hline 6 & $\begin{array}{l}<(0.352,0.608,0.748),(0.018,0.048, \\
0.125),(0.002,0.018,0.09)>\end{array}$ & $1 \rightarrow 2 \rightarrow 5 \rightarrow 6$ \\
\hline
\end{tabular}

Table2. Tabular representation of different triangular fuzzy neutrosophic distance and shortest path.

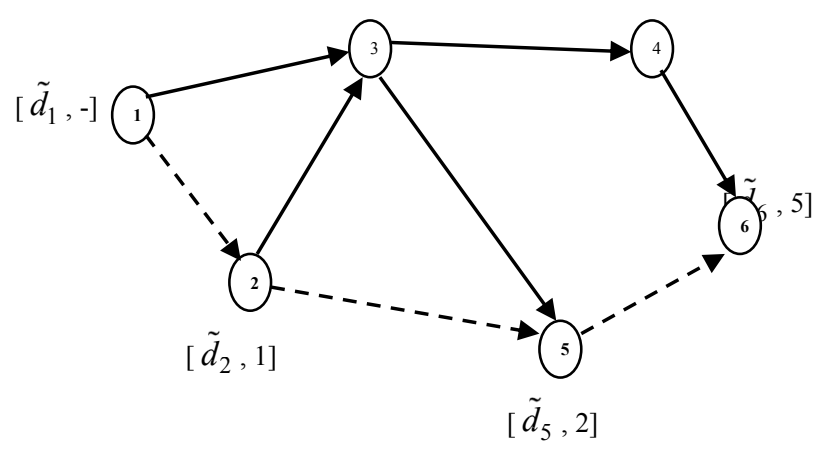

Fig.2. Network with triangular fuzzy neutrosophic shortest distance of each node from node 1

\section{CONCLUSION}

In this paper, an algorithm has been developed for solving shortest path problem on a network where the edges are characterized by triangular fuzzy neutrosophic. We have explained the method by an example with the help of a hypothetical data. Further, we plan to extend the following 
algorithm of triangular fuzzy neutrosophic number shortest path problem in a trapezoidal neutrosophic environment.

\section{Acknowledgment}

The authors are very grateful to the chief editor and reviewers for their comments and suggestions, which is helpful in improving the paper.

\section{REFERENCES}

[1] F. Smarandache, "Neutrosophic set - a generalization of the intuitionistic fuzzy set," Granular Computing, 2006 IEEE International Conference, 2006, p. $38-42$.

[2] F. Smarandache, A unifying field in logic. Neutrosophy: Neutrosphic probability, set, logic,American Research Press, Rehoboth, fouth edition, 2005

[3] F. Smarandache, "A geometric interpretation of the neutrosophic set - A generalization of the intuitionistic fuzzy set," Granular Computing (GrC), 2011 IEEE International Conference, 2011, pp.602606 .

[4] L. Zadeh, Fuzzy sets. Inform and Control, 8, 1965, pp.338-353

[5] K. Atanassov, "Intuitionistic fuzzy sets," Fuzzy Sets and Systems, vol. 20, 1986, pp. 87-96.

[6] H. Wang, F. Smarandache, Y. Zhang, and R. Sunderraman, "Single valued Neutrosophic Sets," Multisspace and Multistructure 4, 2010, pp. $410-413$.

[7] Florentin Smarandache, Neutrosophic Overset, Neutrosophic Underset, and Neutrosophic Offset. Similarly for Neutrosophic Over-/Under-/OffLogic, Probability, and Statistics, 168 p., Pons Editions, Bruxelles, Belgique, 2016; https://arxiv.org/ftp/arxiv/papers/1607/1607.00234.pdf

[8] A. Q. Ansari, R. Biswas \& S. Aggarwal, "Neutrosophication of Fuzzy Models," IEEE Workshop On Computational Intelligence: Theories, Applications and Future Directions (hosted by IIT Kanpur), 14th July 2013.

[9] A. Q. Ansari, R. Biswas \& S. Aggarwal,” Extension to fuzzy logic representation: Moving towards neutrosophic logic - A new laboratory rat," Fuzzy Systems (FUZZ), 2013 IEEE International Conference, 2013, pp.1-8.

[10] G. Garg, K. Bhutani, M. Kumar and S. Aggarwal, "Hybrid model for medical diagnosis using Neutrosophic Cognitive Maps with Genetic Algorithms," FUZZ-IEEE, 2015, 6page.

[11] I. Deli, S. Yusuf, F. Smarandache and M. Ali, Interval valued bipolar neutrosophic sets and their application in pattern recognition, IEEE World Congress on Computational Intelligence 2016.

[12] I. Deli and Y. Subas, A Ranking methods of single valued neutrosophic numbers and its application to multi-attribute decision making problems, International Journal of Machine Learning and Cybernetics, 2016, 1-14.

[13] J. Ye, Single-Valued Neutrosophic Minimum Spanning Tree and Its Clustering Method" Journal of Intelligent Systems 23(3), 2014, pp. 311-324.

[14] J. Ye. Single- valued neutrosophic similarity measures based on cotangent functionand their application in the fault diagnosis of stream turbine. Neural Computing and Applications, 2014. DOI 10.1007/s00521-014-1787-6.

[15] M. Ali, and F. Smarandache, "Complex Neutrosophic Set," Neural Computing and Applications, Vol. 25, 2016, pp.1-18.

[16] Y. Subas, Neutrosophic numbers and their application to multiattribute decision making problems (in Turkish), Master Thesis, 7 Aralk university, Graduate School of Natural and Applied Science, 2015.

[17] R. Chatterje, P.Majumdar, S. K.Samanta, Single Valued neutrosophic multisets, Annals of !fuzzy Mathematics and Informatics, 2015, 15 pages.

[18] P. Biswas, S. Pramanik and B. C. Giri, Cosine Similarity Measure Based Multi-attribute Decision-Making with Trapezoidal fuzzy Neutrosophic numbers, Neutrosophic sets and systems, 8, 2014, pp.47-57.

[19] S. Broumi, F. Smarandache, "New distance and similarity measures of interval neutrosophic sets," Information Fusion (FUSION), 2014 IEEE 17th International Conference, 2014, pp. $1-7$.
[20] P. Biswas, S. Pramanik and B. C. Giri, Aggregation of Triangular Fuzzy Neutrosophic Set Information and its Application to Multiattribute Decision Making, Neutrosophic sets and systems, 12, 2016, pp.20-40.

[21] J. Ye. Trapezoidal fuzzy neutrosophic set and its application to multiple attribute decision making. Soft Computing, 2015. DOI 10.1007/s00500-015-1818-y.

[22] S. Broumi, M. Talea, A. Bakali, F. Smarandache, "Single Valued Neutrosophic Graphs," Journal of New Theory, N 10, 2016, pp. 86-101.

[23] S. Broumi, M. Talea, F. Smarandache and A. Bakali, Single Valued Neutrosophic Graphs: Degree, Order and Size. IEEE World Congress on Computational Intelligence, 2016, pp.2444-2451.

[24] S. Broumi, A. Bakali, M, Talea, and F, Smarandache, Isolated Single Valued Neutrosophic Graphs. Neutrosophic Sets and Systems, Vol. 11, 2016, pp.74-78.

[25] S. Broumi, M. Talea, A. Bakali, F. Smarandache, Interval Valued Neutrosophic Graphs, $\operatorname{SISOM}(2016)$ in press.

[26] S. Broumi, F. Smarandache, M. Talea and A. Bakali, Decision-Making Method Based On the Interval Valued Neutrosophic Graph, Future technologie, 2016, IEEE, In press

[27] S. Broumi, M. Talea, A. Bakali, F. Smarandache, "On Bipolar Single Valued Neutrosophic Graphs," Journal of New Theory, N11, 2016, pp.84-102.

[28] S. Broumi, F. Smarandache, M. Talea and A. Bakali, An Introduction to Bipolar Single Valued Neutrosophic Graph Theory. Applied Mechanics and Materials, vol.841,2016, 184-191.

[29] F. Smarandache," Refined Literal Indeterminacy and the Multiplication Law of Sub-Indeterminacies," Neutrosophic Sets and Systems, Vol. 9, 2015, pp.58.63.

[30] F. Smarandache, "Types of Neutrosophic Graphs and neutrosophic AlgebraicStructures together with their Applications in Technology," seminar, Universitatea Transilvania din Brasov, Facultatea de Design de Produs si Mediu, Brasov, Romania 06 June 2015.

[31] A. Ngoor and M. M. Jabarulla, Multiple labeling Approach For Finding shortest Path with Intuitionstic Fuzzy Arc Length, International Journal of Scientific and Engineering Research,V3, Issue 11, pp.102-106,2012.

[32] S. Okada and T. Soper, " A shortest path problem on a network with fuzzy arc lengths", Fuzzy sets and systems, 109, 2000, pp.129140 .

[33] A. Kumar, and M. Kaur, Solution of fuzzy maximal flow problems using fuzzy linear programming. World Academy of Science and Technology. 87: 28-31, (2011).

[34] S. Majumdar and A. Pal, Shortest Path Problem on Intuitionistic Fuzzy Network, Annals of Pure and Applied Mathematics, Vol. 5, No. 1, November 2013, pp. 26-36.

[35] A. Kumar and M. Kaur, A New Algorithm for Solving Shortest Path Problem on a Network with Imprecise Edge Weight, Applications and Applied Mathematics, Vol. 6, Issue 2, 2011, pp. $602-619$.

[36] A.K. Yadav, B.R. Biswas, On searching fuzzy shortest path in a network, International Journal of Recent Trends in Engineering 2 (3), 2009,pp. 16-18.

[37] P. Jayagowri and G. Geetha Ramani, Using Trapezoidal Intuitionistic Fuzzy Number to Find Optimized Path in a Network, Volume 2014, Advances in Fuzzy Systems, 2014, 6 pages.

[38] F. Hernandes, M. Teresa Lamata, J. L Verdegay, and A. Y. kami, The shortest path problem on networks with fuzzy parameters. Fuzzy Sets and Systems. 158, 2007:pp.1561-1570.

[39] G. Kumar, R. K. Bajaj and N .Gandotra, "Algoritm for shortest path problem in a network with interval valued intuitionstic trapezoidal fuzzy number, Procedia Computer Science 70,2015, pp.123-129.

[40] S. Broumi, A. Bakali, M. Talea and F. Smarandache, Computation of Shortest Path Problem in a Network with Single Valued Neutrosophic Number Based on Ranking Method (submitted).

[41] S. Broumi, A. Bakali, M. Talea and F. Smarandache, Computation of Shortest Path Problem in a Network with SV-trapezoidal neutrosophic numbers, (submitted).

[42] S. Broumi, A. Bakali, M. Talea and F. Smarandache, M. Ali, Shortest Path Problem Under Bipolar Neutrosphic Setting, 2016. In press. 